\title{
Cytauxzoon felis Infection in Domestic Cats, Yunnan Province, China, 2016
}

\section{Feng-Cai Zou, ${ }^{1}$ Zhao Li, ${ }^{1}$ Jian-Fa Yang, Jiang-Yan Chang, Guo-Hua Liu, Yan Lv, Xing-Quan Zhu}

We performed a molecular survey for Cytauxzoon felis infection in 311 domestic cats in Yunnan Province, China, in 2016 and found a prevalence of $21.5 \%$. C. felis infection in domestic and wild cats in other provinces should be investigated to determine parasite prevalence and genetic diversity among cats throughout China.

$\mathrm{C}$ ytauxzoonosis is a tickborne hemoprotozoal disease of both domestic cats and wild felids caused mainly by Cytauxzoon felis protozoa $(1,2)$. In the late $1900 \mathrm{~s}, C$. felis protozoa were reported exclusively in North America, particularly in the mid-Atlantic states of the United States (3), but in the early 2000 s, this pathogen was reported in some countries of South America, and in Europe, several other Cytauxzoon species were identified (4). Cytauxzoonosis of domestic cats has long been considered contagious and deadly (2). However, as research progressed, the virulence of different $C$. felis isolates was found to be inconsistent; some cats were able to survive the infection and potentially serve as natural reservoirs (5).

The number of pet cats around the world is increasing, but the information about the prevalence of $C$. felis infection in domestic cats is limited worldwide. Because of the seriousness of feline cytauxzoonosis and its geographic expansion to more and more regions, informing veterinarians, pet owners, and the general public about this disease has become imperative. The objective of this study was to examine whether $C$. felis infection is present in domestic cats in China.

\section{The Study}

During November-December 2016, we collected whole blood from the femoral vein of 311 domestic cats (74 stray

Author affiliations: Key Laboratory of Veterinary Public Health of Higher Education of Yunnan Province, College of Veterinary Medicine, Yunnan Agricultural University, Kunming, China (F.-C. Zou, Z. Li, J.-F. Yang, J.-Y. Chang, Y. Lv); State Key Laboratory of Veterinary Etiological Biology, Lanzhou Veterinary Research Institute, Chinese Academy of Agricultural Sciences, Lanzhou, China (Z. Li, X.-Q. Zhu); College of Veterinary Medicine, Hunan Agricultural University, Changsha, China (G.-H. Liu)

DOI: https://doi.org/10.3201/eid2502.181182 cats and 237 pet cats) in Yunnan Province in southwestern China using EDTA tubes. We stored these EDTA whole blood samples at $-20^{\circ} \mathrm{C}$ and then performed genomic DNA extraction with the TIANamp Genomic DNA Kit (TianGen, http://www.tiangen.com) following the manufacturer's protocol. To detect $C$. felis infection, we performed a PCR targeting the second internal transcribed spacer (ITS-2) of ribosomal DNA (6). We sequenced amplicons in both directions and compared these sequences with those of other relevant C. felis isolates available in GenBank. We analyzed differences in C. felis prevalence in domestic cats according to lifestyle, region, sex, and age using the $\chi^{2}$ test in SPSS 22.0 standard version for Windows (IBM Corporation, https:// www.ibm.com). We considered differences statistically significant when the $p$ value obtained was $<0.05$.

In total, $67(21.5 \%)$ of 311 examined domestic cats were positive for the $C$. felis protozoan. We sequenced these $C$. felis-positive PCR products and obtained 67 ITS2 sequences; 4 representative sequences were deposited in GenBank (accession nos. MF966369-72). The 67 C. felis ITS-2 sequences shared $98.4 \%-100 \%$ similarity. These sequences had $95.6 \%-100 \%$ similarity with corresponding C. felis ITS-2 sequences available in GenBank.

The prevalence of $C$. felis protozoa in domestic cats in Yunnan Province was 21.5\% (Table), lower than the prevalence in domestic cats in the United States $(30.3 \%, 27 / 89)$ (7) but higher than that in Brazil $(0.66 \%, 1 / 151)(8)$. The $C$. felis prevalence in stray cats $(51.4 \%, 38 / 74)$ was significantly higher $(\mathrm{p}<0.001)$ than that in pet cats $(12.2 \%, 29 / 237)$ (Table), probably because stray cats often live outdoors with poor sanitation, thus having high probability of contact with ticks. However, no significant difference in $C$. felis prevalence was found among domestic cats of different sexes or age groups.

Distinct $C$. felis genotypes of different virulences in domestic cats have been identified, and genetic diversity among $C$. felis populations has been studied by comparisons of $18 \mathrm{~S}$ rRNA, ITS-1, and ITS-2 sequences (1). ITS-1 and ITS-2 rDNA are better genetic markers for assessing $C$. felis genotypic variability ( 9 ) because these sequences evolve faster than the 18S rRNA gene. A combination of ITS-1 and ITS2 sequences has been used to identify the $C$. felis genotypes present in various domestic cats and wild felids (1).

C. felis protozoa are transmitted to domestic cats by ticks, such as Amblyomma americanum and Dermacentor

${ }^{1}$ These authors contributed equally to this article. 
Table. Prevalence of Cytauxzoon felis protozoan in whole blood samples from domestic cats determined by PCR, Yunnan Province, China, 2016

\begin{tabular}{|c|c|c|c|c|}
\hline Variable & No. positive/no. tested & Prevalence, $\%(95 \% \mathrm{Cl})$ & $p$ value & Odds ratio $(95 \% \mathrm{Cl})$ \\
\hline \multicolumn{5}{|l|}{ Lifestyle } \\
\hline Stray & $38 / 74$ & $51.4(40.0-62.7)$ & \multirow[t]{2}{*}{$<0.001$} & $7.6(4.2-13.7)$ \\
\hline Pet & $29 / 237$ & $12.2(8.1-16.4)$ & & Referent \\
\hline \multicolumn{5}{|l|}{ Region } \\
\hline Banna Prefecture & $19 / 38$ & $50.0(34.1-65.9)$ & \multirow[t]{5}{*}{$<0.001$} & $10.8(4.8-24.5)$ \\
\hline Honghe Prefecture & $5 / 15$ & $33.3(9.5-57.2)$ & & $5.4(1.6-17.8)$ \\
\hline Lincang City & $14 / 21$ & $66.7(46.5-86.8)$ & & $21.6(7.6-61.3)$ \\
\hline Kunming City & $16 / 189$ & $8.5(4.5-12.4)$ & & Referent \\
\hline Yuxi City & $13 / 48$ & $27.1(14.5-39.7)$ & & $4.0(1.8-9.1)$ \\
\hline \multicolumn{5}{|l|}{ Sex } \\
\hline $\mathrm{F}$ & 38/172 & $22.1(15.9-28.3)$ & \multirow[t]{2}{*}{0.89} & $1.1(0.6-1.9)$ \\
\hline $\mathrm{M}$ & 29/139 & $20.9(14.1-27.6)$ & & Referent \\
\hline \multicolumn{5}{|l|}{ Age, y } \\
\hline$<5$ & $21 / 83$ & $25.3(15.9-34.7)$ & \multirow[t]{3}{*}{0.18} & $1.8(0.9-3.4)$ \\
\hline $5-10$ & $22 / 133$ & 16.5 (10.2-22.9) & & Referent \\
\hline$>10$ & $24 / 95$ & $25.3(16.5-34.0)$ & & $1.7(0.9-3.3)$ \\
\hline Total & $67 / 311$ & $21.5(17.0-26.1)$ & & \\
\hline
\end{tabular}

variabilis (1). Raising pet cats indoors and preventing and treating ectoparasites of outdoor stray cats would help reduce risk for infection in C. felis protozoa-endemic areas. Some effective antitick insecticides can be used for preventing transmission of this parasite (10).

\section{Conclusions}

Our study revealed a high $(21.5 \%)$ C. felis prevalence in domestic cats in Yunnan Province, China. Further studies are warranted to assess the prevalence of the $C$. felis protozoan in wild felids and domestic cats in other regions of China to estimate its geographic distribution and genetic diversity and to investigate its potential tick vectors.

This study was supported by the Excellent Scientist Fund of Yunnan Agricultural University (grant no. 2015JY03), the Open Funds of the State Key Laboratory of Veterinary Etiological Biology, Lanzhou Veterinary Research Institute, Chinese Academy of Agricultural Sciences (grant no. SKLVEB2017KFKT008), the Elite Program of Chinese Academy of Agricultural Sciences, and the Agricultural Science and Technology Innovation Program (grant no. CAAS-ASTIP-2016-LVRI-03).

\section{About the Author}

Dr. Zou is professor of veterinary parasitology at the Key Laboratory of Veterinary Public Health of Higher Education of Yunnan Province, College of Veterinary Medicine, Yunnan Agricultural University, Kunming, Yunnan, China. His research focuses on the epidemiology and molecular biology of parasitic protozoa and control strategies for parasitic infections of animals and humans.

\section{References}

1. Wang JL, Li TT, Liu GH, Zhu XQ, Yao C. Two tales of Cytauxzoon felis infections in domestic cats. Clin Microbiol Rev. 2017;30: 861-85. http://dx.doi.org/10.1128/CMR.00010-17

2. Sherrill MK, Cohn LA. Cytauxzoonosis: diagnosis and treatment of an emerging disease. J Feline Med Surg. 2015;17:940-8. http://dx.doi.org/10.1177/1098612X15610681
3. Birkenheuer AJ, Le JA, Valenzisi AM, Tucker MD, Levy MG, Breitschwerdt EB. Cytauxzoon felis infection in cats in the mid-Atlantic states: 34 cases (1998-2004). J Am Vet Med Assoc. 2006;228:568-71. http://dx.doi.org/10.2460/ javma.228.4.568

4. Carli E, Trotta M, Chinelli R, Drigo M, Sinigoi L, Tosolini P, et al. Cytauxzoon sp. infection in the first endemic focus described in domestic cats in Europe. Vet Parasitol. 2012;183:343-52. http://dx.doi.org/10.1016/j.vetpar.2011.07.025

5. Rizzi TE, Reichard MV, Cohn LA, Birkenheuer AJ, Taylor JD, Meinkoth JH. Prevalence of Cytauxzoon felis infection in healthy cats from enzootic areas in Arkansas, Missouri, and Oklahoma. Parasit Vectors. 2015;8:13. http://dx.doi.org/10.1186/s13071-014-0618-Z

6. Brown HM, Latimer KS, Erikson LE, Cashwell ME, Britt JO, Peterson DS. Detection of persistent Cytauxzoon felis infection by polymerase chain reaction in three asymptomatic domestic cats. J Vet Diagn Invest. 2008;20:485-8. http://dx.doi.org/ $10.1177 / 104063870802000411$

7. Brown HM, Lockhart JM, Latimer KS, Peterson DS. Identification and genetic characterization of Cytauxzoon felis in asymptomatic domestic cats and bobcats. Vet Parasitol. 2010;172:311-6. http://dx.doi.org/10.1016/j.vetpar.2010.04.041

8. André MR, Herrera HM, de Jesus Fernandes S, de Sousa KCM, Gonçalves LR, Domingos IH, et al. Tick-borne agents in domesticated and stray cats from the city of Campo Grande, state of Mato Grosso do Sul, midwestern Brazil. Ticks Tick Borne Dis. 2015;6:779-86. http://dx.doi.org/10.1016/j.ttbdis.2015.07.004

9. Shock BC, Birkenheuer AJ, Patton LL, Olfenbuttel C, Beringer J, Grove DM, et al. Variation in the ITS-1 and ITS-2 rRNA genomic regions of Cytauxzoon felis from bobcats and pumas in the eastern United States and comparison with sequences from domestic cats. Vet Parasitol. 2012;190:29-35. http://dx.doi.org/10.1016/ j.vetpar.2012.06.010

10. Reichard MV, Thomas JE, Arther RG, Hostetler JA, Raetzel KL, Meinkoth JH, et al. Efficacy of an imidacloprid 10\%/flumethrin $4.5 \%$ collar (Seresto, Bayer) for preventing the transmission of Cytauxzoon felis to domestic cats by Amblyomma americanum. Parasitol Res. 2013; 112(Suppl 1):11-20. http://dx.doi.org/10.1007/ s00436-013-3277-7

Address for correspondence: Xing-Quan Zhu, State Key Laboratory of Veterinary Etiological Biology, Lanzhou Veterinary Research Institute, Chinese Academy of Agricultural Sciences, 1 Xujiaping, Yanchangbu, Lanzhou, Gansu 730046, China; email: xingquanzhu1@hotmail.com 